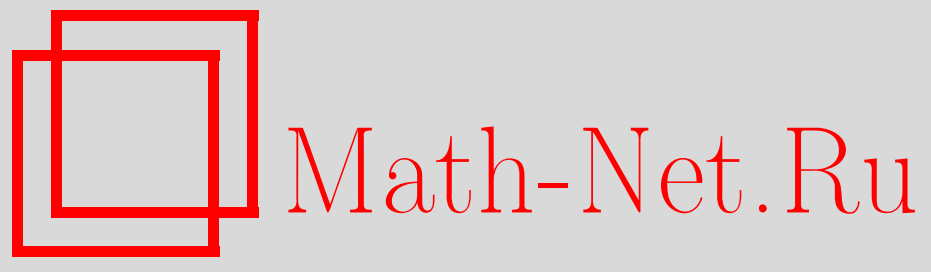

Ю. В. Малыхин, Локальная энтропия в теории обучения, Матем. заметки, 2006, том 80, выпуск 6, 946-949

DOI: https://doi.org/10.4213/mzm3372

Использование Общероссийского математического портала Math-Net.Ru подразумевает, что вы прочитали и согласны с пользовательским соглашением http://www . mathnet.ru/rus/agreement

Параметры загрузки:

IP : 54.224 .135 .184

26 апреля 2023 г., 06:42:45

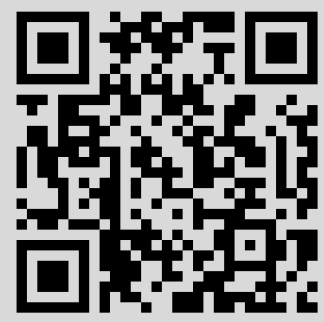




\section{ЛОКАЛЬНАЯ ЭНТРОПИЯ В ТЕОРИИ ОБУЧЕНИЯ}

\section{Ю. В. Малыхин}

1. Обозначения. Если $F(\varepsilon)$ и $G(\varepsilon)$ - функции, определенные при $\varepsilon \in\left(0, \varepsilon_{0}\right)$, то будем писать $F(\varepsilon) \sim G(\varepsilon)$, когда

$$
\lim _{\varepsilon \rightarrow+0} \frac{F(\varepsilon)}{G(\varepsilon)}=1,
$$

и $F(\varepsilon) \asymp G(\varepsilon)$, когда $F(\varepsilon) \leqslant a G(\varepsilon)$ и $G(\varepsilon) \leqslant b F(\varepsilon)$ для некоторых $a, b>0$. Через $\log$ обозначается логарифм по основанию 2 .

2. Постановка задачи теории обучения. Пусть $\rho$ - неизвестная нам вероятностная мера на $X \times Y$. Задача состоит в том, чтобы по выборке $\boldsymbol{z}=\boldsymbol{z}^{(m)}=\left(\left(x_{1}, y_{1}\right), \ldots\right.$, $\left.\left(x_{m}, y_{m}\right)\right)$, состоящей из независимых величин, распределенных по мере $\rho$, аппроксимировать функцию регрессии

$$
f_{\rho}(x)=\int_{Y} y d \rho(y \mid x) .
$$

Здесь $\rho(y \mid x)$ - условная мера. Функция регрессии минимизирует величину ошибки

$$
\mathscr{E}(f)=\int_{X \times Y}(f(x)-y)^{2} d \rho
$$

по всевозможным $f$ - это следует из равенства $\mathscr{E}(f)-\mathscr{E}\left(f_{\rho}\right)=\left\|f-f_{\rho}\right\|_{L_{2}\left(\rho_{X}\right)}^{2}$ (мера $\rho_{X}$ есть проекция меры $\rho$ на $X$, т.е. $\left.\rho_{X}(S):=\rho(S \times Y)\right)$. Таким образом, $f_{\rho}$ наилучшим образом выражает зависимость $y$ от $x$. В данной статье рассматривается следующая ситуация. Во-первых, нам известно распределение величины $X$, т.е. маргинальная мера $\rho_{X}$. Во-вторых, нам известно, что $f_{\rho}$ принадлежит фиксированному классу $\Theta$. В-третьих, функцию регрессии мы будем приближать по норме пространства $L_{2}\left(\rho_{X}\right)$.

Теперь дадим формальное описание задачи. Пусть $X=\mathbb{R}^{d}, Y=[-M, M], Z=X \times Y$. Назовем оценщиком произвольное отображение $E_{m}: \boldsymbol{z} \mapsto f_{\boldsymbol{z}}$, которое по выборке $\boldsymbol{z}=$ $\boldsymbol{z}^{(m)} \in Z^{m}$ строит борелевскую функцию $f_{\boldsymbol{z}}: X \rightarrow Y$. Пусть $\mathscr{M}$ - некоторый класс борелевских вероятностных мер на $Z$. В [1] определяется доверительная функция

$$
\operatorname{AC}_{m}(\mathscr{M}, \eta)=\inf _{E_{m}} \sup _{\rho \in \mathscr{M}} \rho^{m}\left\{\boldsymbol{z}:\left\|f_{\rho}-f_{\boldsymbol{z}}\right\|_{L_{2}\left(\rho_{X}\right)} \geqslant \eta\right\} .
$$

Поскольку, вообще говоря, множество $\left\{\boldsymbol{z}:\left\|f_{\rho}-f_{\boldsymbol{z}}\right\| \geqslant \eta\right\}$ может оказаться неизмеримым относительно меры $\rho^{m}$, под вероятностью понимается внешняя вероятность. Далее, пусть фиксирована борелевская вероятностная мера $\mu$ на $X$ и задано некоторое множество $\Theta$ борелевских функций из $X$ в $Y$. Обозначим через $\mathscr{M}(\Theta, \mu)$ класс тех мер $\rho$, для которых $\rho_{X}=\mu$ и $f_{\rho} \in \Theta$. Таким образом, наша задача состоит в исследовании функции $\mathrm{AC}_{m}(\mathscr{M}(\Theta, \mu), \eta)$.

3. Некоторые определения. Понятно, что чем "массивнее" класс $\Theta$, тем больше будет погрешность оценщиков. Чтобы сформулировать эту зависимость точно, напомним некоторые определения.

Пусть $(S, \tau)$ - метрическое пространство, $A \subset S$. Число элементов в минимальной $\varepsilon$-сети для $A$ в $S$ обозначим через $N_{\varepsilon}(A, S)$. Двоичный логарифм этого числа обозначается через $\mathscr{H}_{\varepsilon}(A, S)$ и называется $\varepsilon$-энтропией $A$ в $S$. Энтропийным поперечником множества $A$ в $S$ называется величина

$$
\varepsilon_{n}(A, S):=\inf \left\{\varepsilon>0: \mathscr{H}_{\varepsilon}(A, S) \leqslant n\right\} .
$$

Работа выполнена при поддержке Российского фонда фундаментальных исследований, грант № 05-01-00066, и Программы поддержки ведущих научных школ, грант № НШ-3004.2003.1.

(C) Ю. В. МАлыхин, 2006 
Через $P_{\varepsilon}$ мы обозначим размер максимальной $\varepsilon$-упаковки в $A$, т.е.

$$
P_{\varepsilon}(A)=P_{\varepsilon}(A, S):=\sup \left\{n: \exists x_{1}, \ldots, x_{n} \in A \tau\left(x_{i}, x_{j}\right) \geqslant \varepsilon\right\} .
$$

Разумеется, $P_{\varepsilon}$ не зависит от объемлющего пространства; иногда $S$ будет служить для указания метрики на $A$.

Кроме этого, нам понадобится немного другой вид энтропии: “локальная" энтропия. Зафиксируем число $c>1$. Определим локальное упаковочное число множества $A$ как

$$
\bar{P}_{\varepsilon}(c, A)=\bar{P}_{\varepsilon}(c, A, S):=\sup \left\{n: \exists x_{1}, \ldots, x_{n} \in A \varepsilon \leqslant \tau\left(x_{i}, x_{j}\right) \leqslant c \varepsilon\right\} .
$$

Данное определение взято из [1]. Впрочем, подобный вид энтропии использовался ранее, cм. [2].

4. Предварительные сведения. Пусть мы находимся в ситуации, описанной в п. 2 . Будем предполагать, что $\Theta$ - компакт в $L_{2}(\mu)$, так что все энтропийные характеристики $\Theta$ конечны. Обозначим

$$
\bar{P}(\varepsilon):=\bar{P}_{\varepsilon}\left(c, \Theta, L_{2}(\mu)\right) .
$$

В работе [1] дана нижняя оценка доверительной функции через локальную энтропию. Прежде чем сформулировать теорему, проведем одно построение. Пусть $Y=[-1,1]$. Рассмотрим набор функций $\left\{f_{i}\right\}_{i=1}^{\bar{P}(\eta)}$, взятый из определения (1). Каждой $f_{i}$ можно сопоставить меру $\rho_{i}$ :

$$
d \rho_{i}(x, y)=\left(\frac{1+f_{i}(x)}{2} d \delta_{1}(y)+\frac{1-f_{i}(x)}{2} d \delta_{-1}(y)\right) d \mu(x),
$$

где $d \delta_{\xi}$ есть дираковская мера единичной массы в $\xi$. Заметим, что так определенные меры лежат в $\mathscr{M}(\Theta, \mu)$, ибо $\left(\rho_{i}\right)_{X}=\mu$ и $f_{\rho_{i}}=f_{i}$.

Теорема [1; теорема 3.1]. Пусть $Y=[-1,1]$. Предположим, что при некотором $\eta>0$ функиии $\left\{f_{i}\right\}, i=1, \ldots, \bar{P}(2 \eta)$, из (1) удовлетворяют условию $\left\|f_{i}\right\|_{C(X)} \leqslant 1 / 4$. Тогда для любого оценщика $f_{\boldsymbol{z}}$ найдется $i \in\{1, \ldots, \bar{P}(2 \eta)\}$ такой, что

$$
\rho_{i}\left\{\boldsymbol{z}:\left\|f_{\boldsymbol{z}}-f_{i}\right\|_{L_{2}(\mu)} \geqslant \eta\right\} \geqslant \min \left(\frac{1}{2},(\bar{P}(2 \eta)-1)^{1 / 2} e^{-8 c^{2} m \eta^{2}-3 / e}\right), \quad m=1,2, \ldots
$$

Верхние же оценки для доверительной функции проводились в терминах обычной энтропии. Наиболее точная теорема содержится в работе [3].

Теорема [3; теорема 1.3]. Предположим, что $\Theta$ удовлетворяет условиям

$$
\varepsilon_{n}\left(\Theta, L_{2}(\mu)\right) \leqslant D n^{-r}, \quad n=1,2, \ldots, \quad\|f\|_{L_{2}(\mu)} \leqslant D \quad \forall f \in \Theta .
$$

Тогда существует оченщик $f_{\boldsymbol{z}}$ такой, что для любой меры $\rho \in \mathscr{M}(\Theta, \mu)$, любого $\eta \geqslant$ $\varepsilon_{0}=C(M, D, r) m^{-r /(1+2 r)}$ и $m \geqslant 240(M / D)^{2}$ выполняется неравенство

$$
\rho^{m}\left\{\boldsymbol{z}:\left\|f_{\boldsymbol{z}}-f_{\rho}\right\|_{L_{2}(\mu)} \geqslant \eta\right\} \leqslant \exp \left(-\frac{m \eta^{2}}{800 M^{2}}\right) .
$$

Оценщик $f_{z}$ в этой теореме строится следующим образом. Рассматривается минимальная $\left(\varepsilon_{0} / 49\right)$-сеть $\mathscr{N}$ в $L_{2}(\mu)$ для множества $\Theta$. Далее полагается

$$
f_{\boldsymbol{z}}:=\arg \min _{f \in \mathscr{N}} \mathscr{E}_{\boldsymbol{z}}(f), \quad \text { где } \quad \mathscr{E}_{\boldsymbol{z}}(f)=\frac{1}{m} \sum_{i=1}^{m}\left(f\left(x_{i}\right)-y_{i}\right)^{2} .
$$

Фактически, в [3] доказывается следующее утверждение, которое мы будем использовать в дальнейшем. 
Лемма. Пусть $\mathscr{N}$ - некоторый набор функиий $X \rightarrow Y, \# \mathscr{N}=N$. Пусть

$$
\min _{f \in \mathscr{N}}\left\|f-f_{\rho}\right\|_{L_{2}\left(\rho_{X}\right)} \leqslant \frac{\eta}{2} .
$$

Тогда для оченщика $f_{\boldsymbol{z}}=\arg \min _{f \in \mathscr{N}} \mathscr{E}_{\boldsymbol{z}}(f)$ выполняется неравенство

$$
\rho^{m}\left\{\boldsymbol{z}:\left\|f_{\boldsymbol{z}}-f_{\rho}\right\|_{L_{2}\left(\rho_{X}\right)} \geqslant \eta\right\} \leqslant(N+1) \exp \left(-\frac{m \eta^{2}}{640 M^{2}}\right) .
$$

ЗАмечАниЕ. Если минимум $\mathscr{E}_{z}(f)$ достигается сразу на нескольких функциях $f$, то мы берем произвольную из них. Таким образом, лемма утверждает, что соответствующее неравенство выполнено для любой функции, минимизирующей $\mathscr{E}_{\boldsymbol{z}}(f)$.

5. Основные результаты. В данной работе получены оценки сверху для доверительной функции через локальную энтропию.

Пусть $c=20$. Таким образом, $\bar{P}(\eta):=\bar{P}_{\eta}\left(20, \Theta, L_{2}(\mu)\right)$. Под $\|\cdot\|$ в данной теореме будем понимать норму пространства $L_{2}(\mu)$.

Teopema. Пyсms

$$
\bar{P}(\eta) \leqslant \varphi(\eta) \quad \forall \eta>0,
$$

причем $\varphi$ не возрастает. Существует ощенщик $f_{z}$ такой, что для любой меры $\rho \in$ $\mathscr{M}(\Theta, \mu)$ и любого $\eta>0$ выполняется неравенство

$$
\rho^{m}\left\{\boldsymbol{z}:\left\|f_{\boldsymbol{z}}-f_{\rho}\right\|>\eta\right\} \leqslant c_{1} \varphi\left(\frac{\eta}{16}\right) \exp \left(-c_{2} m \eta^{2}\right),
$$

где $c_{i}=c_{i}(M)$.

ДокАЗАТЕЛЬСтво. Положим $\eta_{0}=M$ и $\eta_{j}=2^{-j} \eta_{0}$. Выберем минимальный номер $k$ так, что $m \eta_{k}^{2} \leqslant 1$. Пусть $A_{j}$ - максимальные $\left(\eta_{j} / 2\right)$-упаковки в $\Theta$. Обозначим $\bar{A}_{j}:=$ $A_{j} \cap B\left(f_{\rho}, 5 \eta_{j}\right)$, где $B(x, R)$ - замкнутый шар радиуса $R$ с центром в $x$. Заметим, что для любых различных $f, g \in \bar{A}_{j}$ будет выполнена оценка $\eta_{j} / 2 \leqslant\|f-g\| \leqslant 10 \eta_{j}$, откуда $\# \bar{A}_{j} \leqslant \bar{P}\left(\eta_{j} / 2\right)$.

Пусть $\Lambda_{j}-$ множество тех $\boldsymbol{z}$, для которых минимум $\mathscr{E}_{\boldsymbol{z}}$ на множестве $\bar{A}_{j}$ достигается внутри шара $B\left(f_{\rho}, \eta_{j}\right)$. Применяя лемму (роль $\eta$ играет $\eta_{j}$, роль $\left.\mathscr{N}-\bar{A}_{j}\right)$, получаем

$$
\rho^{m}\left(\Lambda_{j}\right) \geqslant 1-\left(\bar{P}\left(\frac{\eta_{j}}{2}\right)+1\right) \exp \left(-\frac{m \eta_{j}^{2}}{640 M^{2}}\right) .
$$

Теперь построим оценщик. Положим $f_{\boldsymbol{z}}^{(0)}:=\arg \min \left\{\mathscr{E}_{\boldsymbol{z}}(f): f \in A_{0}\right\}$. Далее строим оценщики $f_{z}^{(s)}$ по индукции по формуле

$$
f_{\boldsymbol{z}}^{(s+1)}:=\arg \min \left\{\mathscr{E}_{\boldsymbol{z}}(f): f \in A_{s+1} \cap B\left(f_{\boldsymbol{z}}^{(s)}, 3 \eta_{s+1}\right)\right\} .
$$

В качестве окончательного оценщика $f_{\boldsymbol{z}}$ возьмем $f_{z}^{(k)}$.

Докажем по индукции, что при $\boldsymbol{z} \in \Lambda^{(s)}:=\bigcap_{j=0}^{s} \Lambda_{j}$ выполняется неравенство $\| f_{z}^{(s)}-$ $f_{\rho} \| \leqslant \eta_{s}$. Действительно, для $s=0$ это следует из $z \in \Lambda_{0}$. Пусть неравенство выполнено для $s$; докажем его для $s+1$. Из условия $\left\|f_{z}^{(s)}-f_{\rho}\right\| \leqslant \eta_{s}=2 \eta_{s+1}$ следует, что

$$
B\left(f_{\rho}, \eta_{s+1}\right) \subset B\left(f_{z}^{(s)}, 3 \eta_{s+1}\right) \subset B\left(f_{\rho}, 5 \eta_{s+1}\right) .
$$

Поскольку $\boldsymbol{z} \in \Lambda_{s+1}$, минимум $\mathscr{E}_{\boldsymbol{z}}$ на множестве $\bar{A}_{s+1}=A_{s+1} \cap B\left(f_{\rho}, 5 \eta_{s+1}\right)$ достигается внутри шара $B\left(f_{\rho}, \eta_{s+1}\right)$. Значит, этот минимум будет достигаться как раз на $f_{z}^{(s+1)}$. Отсюда $f_{z}^{(s+1)} \in B\left(f_{\rho}, \eta_{s+1}\right)$, что и требовалось доказать.

Возьмем теперь $\eta>0$. При $\eta<4 \eta_{k}$ неравенство (2) очевидно. Пусть $\eta \geqslant 4 \eta_{k}$. Рассмотрим номер $s$ такой, что $\eta / 8<\eta_{s} \leqslant \eta / 4$. Тогда если $z \in \Lambda^{(s)}$, то из (3) получаем

$$
\left\|f_{\boldsymbol{z}}-f_{\rho}\right\| \leqslant\left\|f_{\boldsymbol{z}}^{(s)}-f_{\rho}\right\|+\left\|f_{\boldsymbol{z}}^{(s)}-f_{\boldsymbol{z}}^{(k)}\right\| \leqslant \eta_{s}+3 \eta_{s} \leqslant \eta .
$$


Оценим $\rho^{m}\left(\Lambda^{(s)}\right)$, обозначив для краткости $c_{0}=1 /\left(640 M^{2}\right)$ :

$$
\begin{aligned}
1-\rho^{m}\left(\Lambda^{(s)}\right) & \leqslant\left(\bar{P}\left(\frac{\eta_{s}}{2}\right)+1\right) \exp \left(-c_{0} m \eta_{s}^{2}\right)+\left(\bar{P}\left(\eta_{s}\right)+1\right) \exp \left(-c_{0} m\left(2 \eta_{s}\right)^{2}\right)+\cdots \\
& \leqslant 2 \varphi\left(\frac{\eta}{16}\right) \exp \left(-c_{2} m \eta^{2}\right)+2 \varphi\left(\frac{\eta}{8}\right) \exp \left(-2 c_{2} m \eta^{2}\right)+\cdots \\
& \leqslant 2 \varphi\left(\frac{\eta}{16}\right) \exp \left(-c_{2} m \eta^{2}\right)\left(1+\exp \left(-c_{2} m \eta^{2}\right)+\exp \left(-2 c_{2} m \eta^{2}\right)+\cdots\right) .
\end{aligned}
$$

Остается заметить, что при $\eta \geqslant 4 \eta_{k}$ последний сомножитель ограничен.

6. Некоторые примеры. Для многих классов локальное упаковочное число можно оценить снизу через обычное упаковочное число. В качестве примера приведем лемму из [4].

Лемма [4; лемма 2.1]. Пусть $\Theta-$ компакт в банаховом пространстве В. Предположим, что имеют место оценки

$$
C_{1} \varphi(\varepsilon) \leqslant \log P_{\varepsilon}(\Theta) \leqslant C_{2} \varphi(\varepsilon), \quad \varepsilon \in\left(0, \varepsilon_{1}\right]
$$

с функиией $\varphi(\varepsilon)$, удовлетворяющей следующему условию: для любого $\gamma>0$ существует $A_{\gamma}$ такое, что для всякого $\varepsilon>0$ выполняется неравенство $\varphi\left(A_{\gamma} \varepsilon\right) \leqslant \gamma \varphi(\varepsilon)$. Тогда существуют $c_{1} \geqslant 1 u \varepsilon_{2}>0$ такие, что

$$
\log \bar{P}_{\varepsilon}\left(c_{1}, \Theta\right) \geqslant C_{3} \log P_{\varepsilon}(\Theta), \quad \varepsilon \in\left(0, \varepsilon_{2}\right] .
$$

Приведем два примера множеств, для которых величина $\bar{P}_{\varepsilon}$ существенно меньше $P_{\varepsilon}$.

Первый пример - шар в конечномерном пространстве. Для него $\bar{P}_{\varepsilon}$ ограничено, в то время как $P_{\varepsilon}$ стремится к бесконечности при $\varepsilon \rightarrow 0$.

Второй пример. Зафиксируем число $h>0$. Пусть $A_{h}-$ множество функций $f(z)$, аналитических в полосе $|\operatorname{Im} z|<h$ и периодических с периодом $2 \pi$, для которых при любом $u \in(-h, h)$ выполнено неравенство

$$
\frac{1}{2 \pi} \int_{0}^{2 \pi}|f(t+i u)|^{2} d t \leqslant 1
$$

Данное множество рассмотрим в метрике $L_{2}[0,2 \pi]$, где

$$
\|f\|^{2}=\frac{1}{2 \pi} \int_{0}^{2 \pi}|f(t)|^{2} d t .
$$

(Допуская некоторую вольность, можно считать $A$ подмножеством $L_{2}[0,2 \pi]$.)

Известно, что

$$
\log P_{\varepsilon}\left(A_{h}, L_{2}[0,2 \pi]\right) \sim \frac{2(\log (1 / \varepsilon))^{2}}{h \log e} .
$$

Можно показать, что при фиксированном $c>1$ верна слабая асимптотика

$$
\log \bar{P}_{\varepsilon}\left(c, A_{h}, L_{2}[0,2 \pi]\right) \asymp \log \frac{1}{\varepsilon} .
$$

\section{СПИСОК ЦИТИРОВАННОЙ ЛИТЕРАТУРЫ}

[1] R. DeVore, G. Kerkyacharian, D. Picard, V. Temlyakov, Mathematical methods for supervised learning, IMI Preprints, 22, 2004. [2] Y. Yang, A. Barron, Ann. of Statist., 27:5 (1999), 1564-1599. [3] V. Temlyakov, Approximation in learning theory, IMI Preprints, 5, 2005. [4] V. Temlyakov, Optimal estimators in learning theory, IMI Preprints, 23, 2004.

Ю. В. Малыхин

Поступило

Московский государственный университет

23.03.2006

им. М. В. Ломоносова

Исправленный вариант 11.04.2006 